\author{
KULLIYYAH OF MEDICINE \\ INTERNATIONAL ISLAMIC UNIVERSITY MALAYSIA
}

RESEARCH PROPOSAL

\title{
HIGH-MOBILITY GROUP BOX 1 (HMGB1) PROTEIN EVALUATION IN MPTP- INDUCED ZEBRAFISH MODEL OF PARKINSON'S DISEASE
}

Khairiah Razali ${ }^{1}$, Noratikah Othman ${ }^{2}$, Mohd Hamzah Mohd Nasir ${ }^{3}$, Abd Almonem Doolaanea $^{4}$, Jaya Kumar ${ }^{5}$, and Wael M. Y. Mohamed1, 1,6*

${ }^{1}$ Department of Basic Medical Sciences, Kulliyyah of Medicine, International Islamic University Malaysia (IIUM), Kuantan, Malaysia, ${ }^{2}$ Department of Basic Medical Sciences, Kulliyyah of Nursing, International Islamic University Malaysia (IIUM), Kuantan, Malaysia, ${ }^{3}$ Central Research and Animal Facility (CREAM), Kulliyyah of Science, International Islamic University Malaysia (IIUM), Kuantan, Malaysia, ${ }^{4}$ Department of Pharmaceutical Technology, Kulliyyah of Pharmacy, International Islamic University Malaysia (IIUM), Kuantan, Malaysia, ${ }^{5}$ Department of Physiology, Faculty of Medicine, UKM Medical Centre (UKMMC), Kuala Lumpur, Malaysia, ${ }^{6}$ Clinical Pharmacology Department, Menoufia Medical School, Menoufia University, Menoufia, Egypt

*Correspondence:

Wael M. Y. Mohamed

wmy107@gmail.com 


\section{INTRODUCTION}

\subsection{Study background}

Parkinson's disease (PD) is a progressive neurodegenerative disease that attacks dopaminergic neurons in substantia nigra pars compacta $(\mathrm{SNpc})$, a brain region that is typically involved in motor control (DeMaagd \& Philip, 2015; Harris et al., 2020). According to the 2015 Global Burden of Disease, the prevalence and death rates of PD had increased significantly over the past 25 years and currently, there are more than 10 million reported PD cases worldwide (Feigin et al., 2017; Ray Dorsey et al., 2018). Parkinson's disease affects the quality of life as it causes movement impairments as well as deteriorates psychological and cognitive functioning (Yang et al., 2016; Maiti et al., 2017). The main hallmarks of PD pathology are substantial loss of dopaminergic (DA) neurons in SNpc and intracellular aggregation of $\alpha$-synuclein protein or also known as Lewy bodies (LBs) formation (Stefanis, 2012; Barnhill et al., 2020). In molecular context, PD is often associated with mitochondrial dysfunctionality, oxidative stress and chronic neuroinflammation, all of which are believed to trigger subsequent neurodegeneration. At the moment, there is no cure to this disease and researchers are still overwhelmed by its complexity, despite extensive studies have been done.

When neuronal homeostasis is disturbed, the central nervous system (CNS) elicits a defence mechanism in the form of inflammatory response to counter the disturbance. Although it is neuroprotective, however, prolonged inflammation can cause detrimental effects to the neurons (Morales et al., 2016; Sochocka et al., 2017). Chronic neuroinflammation is believed to be one of the biggest factors that contribute to the pathogenesis of PD (Wang et al., 2015). High-mobility group box 1 (HMGB1) protein, or also known as amphoterin, is a DNA-binding nuclear protein that in normal condition, plays a role in nucleosome structural maintenance and transcription regulation (Magna \& Pisetsky, 2014; Paudel et al., 2018). Nevertheless, in pathologic condition, HMGB1 is released to the extracellular matrix as damaged-associated 
molecular protein (DAMP) and triggers downstream inflammatory response through binding with RAGE (receptor for advanced glycation end-products) and toll-like receptors (TLRs) (Santoro et al., 2016). HMGB1 has been implicated in PD due to its involvement with chronic neuroinflammation, oxidative stress, autophagy disorders and consequently, neuronal apoptosis (Angelopoulou et al., 2018). In an animal study of PD, HMGB1 was shown to be upregulated following neurotoxin induction and caused dopaminergic neuronal death through inducing neuroinflammation, exacerbating $\alpha$-synuclein aggregation and disturbing autophagy process (Huang et al., 2017). Inhibition of HMGB1 on the other hand, was reported to suppress inflammatory activity and protect dopaminergic neurons against degeneration (Sasaki et al., 2016). With current evidences, HMGB1 has emerged as a novel target for the treatment of PD. Though proven to be involved in PD pathogenesis, but its molecular mechanism is not yet fully understood.

Since decades ago, zebrafish has been an ideal animal model for PD molecular studies due to its close homology to human (Shehwana \& Konu, 2019) and easy genetic manipulations (Koster \& Sassen, 2015). Zebrafish also expresses HMGB1, which is involved in forebrain development (Zhao et al., 2011), and like humans, in immune response mechanism (Fang et al., 2014). Since the function of this protein is conserved in both species and zebrafish is suitable to be used for PD molecular studies, this provides a promising platform in investigating the involvement of HMGB1 in PD pathogenesis. By using anti-sense HMGB1 morpholino oligonucleotides to knockdown HMGB1 expression and 1-methyl-4-phenyl-1,2,3,6tetrahydropyridine (MPTP) neurotoxin to induce parkinsonism in adult zebrafish, this current study aims to delineate the role of HMGB1 protein in MPTP-induced zebrafish model of PD. Findings from this study will pave the way in designing potential therapies targeting molecular docking for the inhibition of HMGB1 protein. In longer term, this study will help in addressing 
fundamental role of HMGB1 as a novel target for PD that may open the venue for proper treatment of this chronic progressive neurodegenerative disease.

\subsection{Problem statement}

Molecular studies have proven beneficial in understanding the pathophysiology of PD. Besides, advancing application of zebrafish as PD model have enabled researchers to conduct molecular studies with more promising outcomes and significance. Current evidences reported on the association of HMGB1 protein with neuroinflammation-induced PD pathogenesis (Sasaki et al., 2016; Huang et al., 2017; Angelopoulou et al., 2018). However, to the extent of our knowledge, the molecular mechanism pertaining to HMGB1 involvement in PD are still elusive.

Hence, we propose to conduct a study on HMGB1 protein, to elucidate its role in the pathogenesis of MPTP-induced zebrafish model of PD. This study will answer the question pertaining to the involvement of HMGB1 in PD development and whether the knockdown of this protein can improve PD symptoms in zebrafish, particularly MPTP-induced motility disorders.

\subsection{General objective}

To investigate the role of HMGB1 in the development of parkinsonian motility disorders using MPTP-induced zebrafish model of PD.

\subsection{General hypothesis}

HMGB1 is involved in the development of parkinsonian motility disorders of MPTP-induced zebrafish model of PD by mediating chronic neuroinflammatory response. 


\subsection{Specific objectives}

1.5.1. To establish and validate zebrafish model of PD via intraperitoneal injection of MPTP neurotoxin.

1.5.2. To knockdown the expression of HMGB1 in the neurons of zebrafish brain using anti-sense HMGB1 morpholino oligonucleotides.

1.5.3. To evaluate the effect of HMGB1 knockdown at behavioural, histopathological, and molecular levels.

\section{LITERATURE REVIEW}

\subsection{Parkinson's disease aetiology and pathophysiology}

Parkinson's disease affects the dopaminergic neuronal population, particularly in SNpc. Since the SNpc is the origin of nigrostriatal dopaminergic system, gradual loss of DA neurons in this brain region will perturb dopaminergic neuronal projection to the striatum and result in subsequent loss of dopamine input (Sarath Babu et al., 2016; Barnhill et al., 2020). Striatum, apart from the SNpc, is mainly associated with movement regulations, which is why PD is often linked to motility disorders. Apart from substantial dopamine loss, another hallmarks of PD pathology are $\alpha$-synuclein protein aggregations inside neuronal cells, or also known as Lewy bodies (LBs) formation (Stefanis, 2012; Barnhill et al., 2020), as well as chronic neuroinflammation (Troncoso-Escudero et al., 2018). The former causes toxicity to the neurons thus triggers neuronal damage (Meade et al., 2019) while the latter is the result of increased DAMPs population and other pro-inflammatory components (Bajwa et al., 2019). Besides that, molecular studies on PD also revealed mitochondrial dysfunctionality inside the brain. PD patients were reported to have deficient level of mitochondrial complex I, an enzyme that is responsible for the initiation of mitochondrial respiratory chain (Mimaki et al., 2012). Significant loss of mitochondrial complex I leads to failure in ATP synthesis and increased 
production of reactive oxygen species (ROS), which subsequently contributes to the death of DA neurons (Kouli et al., 2018).

Although the absolute cause of PD is still largely unknown, this disease is regarded as a multifactorial disease because it is believed to be influenced by both environmental and genetic factors. Increased longevity is the most known aetiology of PD and it is considered to be the biggest contributing factor to the development of this disease (Hindle, 2010; Reeve et al.,2014; Ball et al., 2019). In fact, only four per cent of reported PD cases came from individuals under the age of 50 (Lee et al., 2019). Other than that, some known synthetic compounds are also evidenced to possess neurotoxin properties that can induce PD-like symptoms in humans, such as MPTP, 6-hydroxydopamine (6-OHDA), as well as several herbicides and pesticides like rotenone and paraquat. In the context of genetic factor, researchers identified several mutations to certain genes that can lead to either autosomal dominant or recessive PD development. For example, mutated snca and lrrk2 gene particularly causes autosomal dominant PD while mutated prkn or pinkl gene triggers the development of autosomal recessive PD (Billingsley et al., 2018; Pang et al., 2019). In terms of inheritance, only a small percentage of individuals diagnosed with PD has a family history of PD symptoms. According to Tran et al. (2020), more than $85 \%$ of PD cases are reported to be sporadic, or in other words, not inherited. From the above findings, it can therefore be concluded that, since PD is a multifactorial disease and majority of PD cases are sporadic, it is definitely challenging to find the cure to completely treat this disease.

As previously mentioned, PD is often linked to motor impairments, hence the main pathology of PD is progressive deterioration of movement ability. Individuals diagnosed with PD are usually presented with symptoms such as resting tremor (tremor during relaxation), bradykinesia (slowness of movement), dyskinesia (uncontrolled, involuntary movements), postural imbalance, and muscular stiffness (Moustafa et al., 2019; Cilia et al., 2020). Besides 
motor symptoms, PD patients can also develop non-motor symptoms, which typically involve psychological and cognitive functioning, for example, executive dysfunction, working memory impairment, sleep dysregulation and psychiatric disturbances (Hermanowicz et al., 2019; Peball et al., 2020). Having said that, as this disease progresses, PD does not only restrict locomotor activity but it also influences mental health, all of which can severely affect the quality of life.

Despite extensive studies, there is still no available treatment that can cure PD completely. Current management involve giving patients medications that can only elevate motor symptoms without actually curing it. The most popular medication for PD is levodopa, a dopamine precursor that helps in replenishing dopamine population in the brain. Levodopa improves motor functions and slows down the progression of PD but at the same time elicit adverse side effects to the patients. When the disease progresses and patients need higher dose of levodopa to elevate tremors, this increases the chance of them experiencing hallucinations and delusions (Chen, 2017). Few other available PD medications include dopamine agonists and monoamine oxidases (MAO) inhibitors, which are also used to restore dopamine level and dopaminergic neurotransmission in the brain (Krishna et al. , 2014; Hajj, 2018). In some cases where patients respond poorly to medications, it is suggestive to do deep brain stimulation (DBS, if they are deemed fit) in which abnormal motor signals are interrupted by external electrical impulses (Hickey \& Stacy, 2016; Sveinbjornsdottir, 2016). However, similar to other medications, DBS can only alleviate uncontrolled tremors without actually curing the dopamine loss.

In view of all that has been mentioned so far, accumulating evidences understood the underlying pathophysiological mechanisms of PD, which include neuroinflammation, mitochondrial dysfunctions in dopaminergic neurons, $\alpha$-synuclein aggregations and autophagy 
disturbances, however, the molecular changes in each of these pathophysiological perturbations are still largely inclusive.

\subsection{High-mobility group box 1 (HMGB1) protein}

Several studies thus far have linked chronic neuroinflammation with PD development (Sasaki et al., 2016; Huang et al., 2017; Angelopoulou et al., 2018). High-mobility group box 1 protein is a DNA-binding nuclear protein that is actively secreted following cytokine stimulation and passively released during cell death or insult (Angelopoulou et al., 2018). In normal condition, HMGB1 is expressed inside the nucleus of a cell playing its role as architectural binding factor and transcription regulator. However, in a condition where cells are damaged or insulted, HMGB1 is released as DAMPs from the nucleus into the cytoplasm and subsequently to the extracellular matrix. HMGB1 acts as alarmin, a danger signal capable of stimulating the immune system to elicit inflammatory response (Frank et al., 2015). It has been demonstrated that following an immune response, HMGB1 can be secreted by degenerating or damaged neurons, as well as by various immune cells such as macrophages, monocytes, and natural killer (NK) cells (Yang et al., 2013). Overexpression of HMGB1 results in uncontrolled neuroinflammation, that will eventually lead to cell damage and cell death.

Analysis on post-mortem human PD brain indicated that HMGB1 level is upregulated in the substantia nigra as well as the cerebrospinal fluid and serum (Santoro et al., 2016). Following PD development, dysfunctions of several mechanisms such as mitochondrial respiratory chain and protein autophagy as previously mentioned, cause continuous neuronal insults, thus, more HMGB1 proteins are being secreted from inflammatory cells to perform its function as alarmin. When this happens over a prolonged time, it will result in chronic neuroinflammation, which will potentially worsen the disease even more. Gao et al. (2011) investigated on inflammation-induced PD when dopaminergic neurons are co-cultured with 
microglial cells. The study revealed that the presence of microglial cells with dopaminergic neuronal culture upregulates nuclear factor- $\mathrm{B}(\mathrm{NF} \kappa \mathrm{B})$ to stimulate downstream inflammatory response and trigger neurodegeneration. Interestingly, such observations were not seen when HMGB1 activity is neutralized, which may suggest probable involvement of HMGB1 protein in the heightened risk of developing PD (Gao et al., 2011).

In a more current study, Lee et al. (2014) in their thorough analysis were able to show that HMGB1 provokes microglia-mediated neuroinflammation and suppression of this protein exerts neuroprotective effect on the neurons. Consistent with above findings, neutralization of HMGB1 expression inhibits dopaminergic neuronal loss in an MPTP-induced rodent model of PD (Santoro et al., 2016). Collectively, there seems to be some evidence to indicate that although beneficial at some level, HMGB1 plays a detrimental role in the immune response that may lead to the progression of PD. Therefore, this protein represents a promising target in the future treatment of PD that needs to be carefully investigated. Whilst some research has been carried out on the mediation of HMGB1 on neuroinflammatory process, the mechanism by which it is involved in PD development has not yet been well established.

\subsection{MPTP-induced zebrafish as PD model}

The past three to four decades have witnessed astounding development on zebrafish application in scientific research. This species is an ideal model for the study of the nervous system due to its conserved homology and synteny with human (Barbazuk et al., 2000; Howe et al., 2013). Ever since its establishment as a model organism in late 1900s, zebrafish has been used in various research on neurodegenerative diseases, including PD, Alzheimer's disease, and Huntington's disease (Freeman et al., 2007; Eisen, 2020). Parkinson's disease affects the production of dopaminergic neurons in the substantia nigra pars compacta (SNpc) and striatum. In zebrafish, the equivalences of these two regions are posterior tuberculum of ventral 
diencephalon (vDn) and ventral telencephalon (vTn), respectively (Figure 1) (Correia et al., 2017; Vijayanathan et al., 2017).

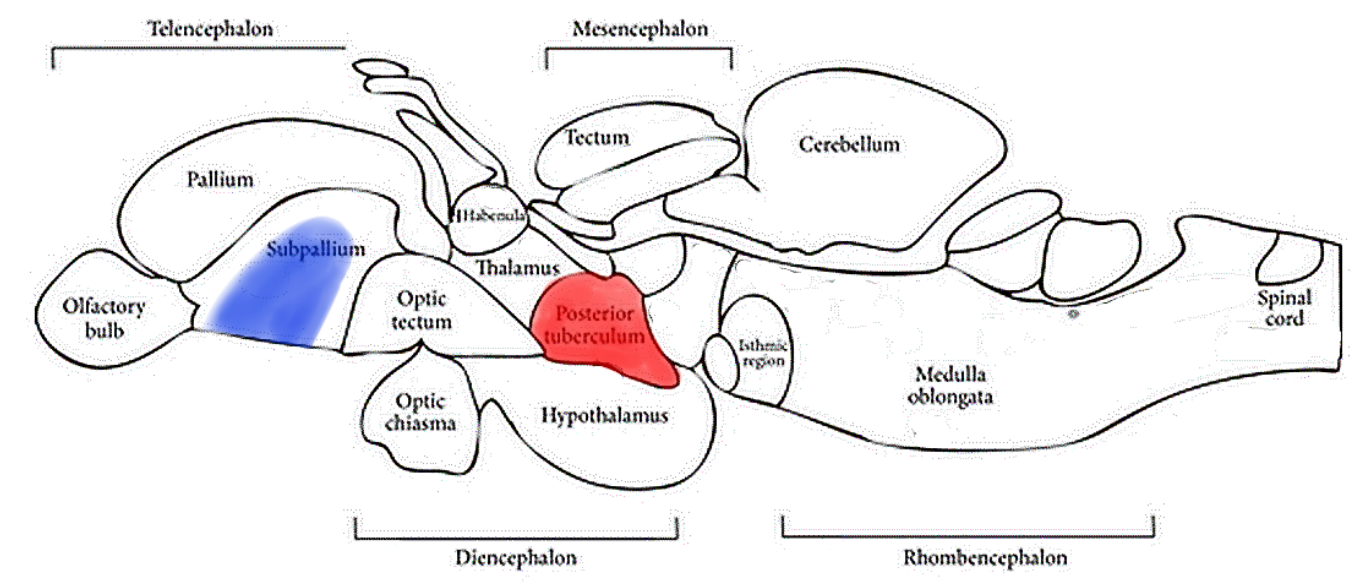

Figure 1 Illustration of the zebrafish brain in sagittal view. Posterior tuberculum of ventral diencephalon (red) and ventral telencephalon (blue) are equivalent to human SNpc and striatum, respectively (adapted from Toledo-Ibarra et al., 2013).

1-methyl-4-phenyl-1,2,3,6-tetrahydropyridine (MPTP) is the most widely used neurotoxin to simulate PD in zebrafish. This compound is a dopaminergic neurotoxin that was accidentally discovered in 1980s when a number of drug users developed acute PD-like symptoms upon injecting themselves with MPTP-containing heroin (Stepens \& Taba, 2016; Langston, 2017). It was then found that besides human, MPTP is able to trigger parkinsonism in other animal species like primates, rodents, and zebrafish as well (Vaz et al., 2018). Animal models of MPTP-induced PD were then developed to meet research demand of studying PD.

As noted by Barnhill et al. (2020), zebrafish metabolizes MPTP in a similar way as human. MPTP easily crosses the blood brain barrier (BBB) into the CNS and is taken up by glial cells (Zeng et al., 2018). Inside the glial cells, MPTP is metabolized by MAO B enzyme to its active form, 1-methyl-4-phenylpyridinium (MPP+) (Bajpai et al., 2013; Robea et al., 2020). Structurally, MPP+ is analogous to dopamine (Fernagut, 2016), hence, it is readily 
transported into dopaminergic neurons by dopamine transporter (DAT) that is located at the presynaptic membrane. MPP+ inside the neurons is toxic as it concentrates around mitochondria and disturbs mitochondrial respiratory chain by inhibiting the action of mitochondrial complex I enzyme (Zawada et al., 2011). This action then results in mitochondrial dysfunction and oxidative stress (Perier \& Vila, 2012, Robea et al., 2020), which consequently leads to dopaminergic neuronal death, closely mimicking PD pathology.

Following MPTP administration, adult zebrafish displays behavioural alterations such as reduced swimming speed and aberrant swimming behaviour, which are reflected as bradykinesia in humans. In addition, MPTP-induced zebrafish also exhibit increased freezing bouts and poor response to tactile stimuli (Lam et al., 2005; Wasel \& Freeman, 2020), indicating significant reduction in locomotor activities and sensory deficit. Weakened touch sensory was also seen following neurotoxin administration in rodent model of PD, which is thought to be influenced by striatal dysfunction due to reduction of striatal dopamine population (Ketzef et al., 2017). Based on the abovementioned findings, the genetic similarity of zebrafish to human and its relatable behaviours have conclusively been shown that MPTPinduced zebrafish is a genetically and phenotypically ideal model to be used in investigating PD pathology in both molecular and behavioural levels.

\subsection{Significance of current study}

Despite extensive research has been carried out investigating the hallmarks of PD pathophysiology, most studies in this area have only focused on the pathology and possible aetiology that lead to the neuro-behavioural alterations. However, there is still very little scientific understanding pertaining to the molecular mechanism of actions that influence the observed pathology and changes. Since chronic neuroinflammation is one of the hallmarks of PD pathogenesis and HMGB1 is believed to be involved in mediating inflammatory response, 
hence, investigating the role of HMGB1 and what lies in between HMGB1/neuroinflammation activation pathway and PD development are highly reasonable to be put as major discussion.

Therefore, the main aim of this study is to evaluate the role of HMGB1 protein in the development of PD, especially in terms of motility disorders, using MPTP-induced zebrafish as model organism. At the end of this study, it is hoped that the functional role of HMGB1 in mediating PD development can be well evaluated. By providing experimental evidence on the role imposed by HMGB1 in inflammatory response mechanism, knowledge gap between chronic neuroinflammation and PD pathogenesis can be eliminated. In longer term, future studies can benefit from this finding in the quest of uncovering promising treatment or discovering drugs that may improve motility disorders and potentially alleviate PD completely.

\section{METHODOLOGY}

\subsection{Animal subject and husbandry}

Adult wild type $\mathrm{AB}$ zebrafish (Danio rerio) both male and female with body length of approximately 3-4 cm will be used in this study. All zebrafish will be bred and housed in 9 litre acrylic tanks located at the Central Research and Animal Facility (CREAM), International Islamic University Malaysia (Kuantan Campus), Pahang, Malaysia. Each tank will consist of 30-35 adult zebrafish with mixed sex groups and they will be fed with dry feed diet (Zeigler Bros Inc, PA, USA) twice per day. The water inside the tanks will be filtered and equipped with air pump to ensure sufficient oxygenation, and the housing systems will be kept maintained at $26-28^{\circ} \mathrm{C}$ and $\mathrm{pH}$ level of $6.5-7.5$, with $14: 10 \mathrm{~h}$ light and dark cycle (Benchoula et al., 2019; Murugesu et al., 2019).

Prior to the start of the experiment, all zebrafish will be monitored and maintained in standard conditions to ensure that they are in good health and are not distressed. Zebrafish exhibits shoaling behaviour and aggression in stressful condition (Aleström et al., 2020; 
Kleinhappel et al., 2019). Any stress inducers will be avoided by minimizing changes in water composition and quality (such as temperature, salinity, $\mathrm{pH}$ level and oxygen content) as well as light intensity. All considerations will be taken to minimize zebrafish suffering and number of zebrafish necessary.

\subsection{Experimental design}

(Corresponding to Specific Objective 1)

\subsubsection{Induction of MPTP on zebrafish} 1-methyl-4-phenyl-1,2,3,6-tetrahydropyridine (MPTP) is a neurotoxin compound capable of inducing PD-like symptoms in zebrafish. Powdered MPTP hydrochloride manufactured by Sigma-Aldrich, MO, USA (Cat\# M0896) will be purchased and a fresh working solution $(10 \mu \mathrm{g} / \mu \mathrm{L})$ will be prepared by dissolving the powder in sterile water (Sarath Babu et al., 2016). Two doses of $50 \mu \mathrm{g}$ MPTP working solution will be injected into the zebrafish via intraperitoneal injection (i/p) with 24h interval between doses (total MPTP dose will be $100 \mu \mathrm{g})$. According to Sarath Babu et al. (2016), one dose of $50 \mu \mathrm{g}$ per day is adequate to exert parkinsonism effect and does not cause lethality to zebrafish. Sterile water will be administered to control group. Prior to the $\mathrm{i} / \mathrm{p}$ injection, zebrafish will be anesthetized in $0.1 \%$ tricaine solution. The entire procedure will be done accordingly and zebrafish will be monitored upon recovery for any side effects or injuries.

\subsubsection{Validation of MPTP-induced zebrafish model}

The validation procedure will be done following the methods described by Sarath Babu et al. (2016), which include behavioural, gene expression (real-time-qPCR), and protein (western blotting) analyses. 


\subsubsection{Behavioural analysis}

After 24h following MPTP induction, zebrafish will be transferred into a transparent 3 litre tank to observe their behaviour. Cameras will be mounted on top and in front of the tank to record parameters such as freezing duration (in sec), number of freezing bouts, distance travelled (in $\mathrm{mm}$ ), and swimming speed (in $\mathrm{mm} / \mathrm{s}$ ). Each zebrafish will be allowed to swim in the tank for 2 mins and its behaviour will be recorded throughout the swimming period (Sarath Babu et al., 2016). Recorded videos will be evaluated and measured using EthoVision software (Noldus Information Technology, NL). All parameters will be statistically analysed to compare the behaviours between MPTP-induced and control groups.

\subsubsection{Gene expression analysis using RT-qPCR}

RT-qPCR assay will be conducted to quantify and compare targeted gene expressions between control and MPTP-induced zebrafish. Zebrafish will be anesthetized with $0.1 \%$ tricaine solution prior to harvesting the brain. Harvested brain tissues from MPTP-induced and control groups will be rinsed with isotonic solution and prepared for total RNA extraction using TRIzol reagent (Invitrogen, CA, USA; Cat\# 15596026). Subsequently, cDNA synthesis will be performed using reverse transcriptase following manufacturer's protocol (SuperScript III; Invitrogen, CA, USA; Cat\# 11732020). RT-qPCR will be conducted using specific primers (Table 1) to analyse several PD-associated genes including lrrk2, park2, park7, sncga and sncgb genes. $\beta$-actin and GAPDH genes will be used as internal controls. Quantitative comparison will be made between MPTP-induced and control groups to estimate relative expression of the selected genes. 
Table $1 \quad$ Forward and reverse primers for each target gene.

\begin{tabular}{|c|l|l|l|}
\hline No & Target gene & Forward primer & Reverse primer \\
\hline 1. & $\begin{array}{l}\text { lrrk2 (Leucine-rich repeat kinase } \\
2 \text { ) }\end{array}$ & $\begin{array}{l}\text { ACTCGGATTAAGTT } \\
\text { CCACCAGA }\end{array}$ & $\begin{array}{l}\text { CAGTGAGGGTTGAT } \\
\text { GGTCTGTA }\end{array}$ \\
\hline 2. & $\begin{array}{l}\text { park2 (Parkin RBR E3 ubiquitin } \\
\text { protein ligase) }\end{array}$ & $\begin{array}{l}\text { ACAGACATCATGAC } \\
\text { TCCAGTGC }\end{array}$ & $\begin{array}{l}\text { ACACGGAAATGAT } \\
\text { GAACCTCTT }\end{array}$ \\
\hline 3. & $\begin{array}{l}\text { park7 (Parkinson protein 7, DJ- } \\
1)\end{array}$ & $\begin{array}{l}\text { GAAAGAGGTGTTG } \\
\text { AAGGACCAG }\end{array}$ & $\begin{array}{l}\text { TGATCACGTTACCA } \\
\text { TCCTTCTG }\end{array}$ \\
\hline 4. & sncga (Synuclein gamma a) & $\begin{array}{l}\text { ATGCACTGAAGAA } \\
\text { GGGATTCTC }\end{array}$ & $\begin{array}{l}\text { AGATTTGCCTGGTC } \\
\text { AGTTGTTT }\end{array}$ \\
\hline 5. & sncgb (Synuclein gamma b) & $\begin{array}{l}\text { GACTAAAGCTGGG } \\
\text { GTTGAAGAG }\end{array}$ & $\begin{array}{l}\text { CGTTCTCCAGTCCC } \\
\text { TCTACTGT }\end{array}$ \\
\hline 6. & $\beta$-actin & $\begin{array}{l}\text { GCCGGGACCTGACT } \\
\text { GACTAC }\end{array}$ & $\begin{array}{l}\text { TTCTCCTTAATGTC } \\
\text { ACGCACGAT }\end{array}$ \\
\hline 7. & $\begin{array}{l}\text { GAPDH (Glyceraldehyde-3- } \\
\text { phosphate dehydrogenase) }\end{array}$ & $\begin{array}{l}\text { ACCCACTCCTCCAC } \\
\text { CTTTGAC }\end{array}$ & $\begin{array}{l}\text { TGTTGCTGTAGCCA } \\
\text { AATTCGTT }\end{array}$ \\
\hline
\end{tabular}

3.2.2.3. Protein expression analysis using western blotting

Western blotting analysis will be performed to validate and compare the expression of targeted proteins between control and MPTP-induced zebrafish. Total proteins will be extracted from the pooled brain tissue samples using radioimmunoprecipitation assay (RIPA) lysis buffer (Thermo Fisher Scientific, MA, USA; Cat\# 89900) with sonication for homogenization. Extracted total proteins will be quantified using bicinchoninic acid assay (BSA assay) before proceeding to the next step.

$50 \mu \mathrm{g}$ of the total protein from each group will be gel electrophoresed in SDS-PAGE and consequently transferred to polyvinylidene fluoride (PVDF) membrane via electroblotting. Following that, membrane will be incubated with the following primary antibodies: anti- $\alpha-$ synuclein antibody (1:1000; Cell Signaling Technology, MA, USA; Cat\# 4179), anti-tyrosine hydroxylase antibody (TH; 1:1000; Merck Millipore, MA, USA; Cat\# MAB318), antiHMGB1 antibody (1:500; Abcam, UK; Cat\# ab233236), anti-Iba1 antibody (1:100; Wako Pure Chemicals Industries, Germany; Cat\# 019-19741) and anti-NFkB p65 antibody (1:1000; Novus 
Biologicals, CO, USA; Cat\# NBP2-67352) at respective optimum dilution for 1 to 2 hours at room temperature. $\beta$-tubulin expression will be used as internal control. Next, the membrane will be incubated with their respective HRP-conjugated secondary antibody and processed following manufacturer's protocol for the chemiluminescent detection and quantification.

(Corresponding to Objective 2)

\subsubsection{HMGB1 knockdown using morpholino} oligonucleotides

Morpholino oligonucleotides (MOs) are synthetic oligonucleotides used to inhibit RNA transcripts from being translated to proteins (Yuan et al., 2013). HMGB1 anti-sense MOs (5'GATCCTTCCCCATCTTTGCCTAAAT-3') will be utilized in this study to knockdown the expression of HMGB1 protein. In addition, a standard control MOs (5'CCTCTTACCTCAGTTACAATTTATA-3') will be used as negative control (Fang et al., 2014).

Zebrafish will be first anesthetized with $0.1 \%$ tricaine solution. Then, they will be carefully fixed with forceps and a small incision will be created into the skull using a needle. Zebrafish will be microinjected with $1 \mu 1$ of aliquot containing $500 \mu \mathrm{M}$ morpholino solution mixed with fluorescent dye (for confirmation of MOs delivery) using a microinjection capillary oriented towards the ventral diencephalon (vDn). To reach the target region, the microcapillary will be set to an injection angle and depth of $60^{\circ}$ and $1200 \mu \mathrm{m}$, respectively (Vijayanathan et al., 2017).

Validation process will be done through western blotting (to quantify HMGB1 protein expression) and immunohistochemistry (to validate the location of HMGB1 knockdown) analyses. 12 hours after confirmation, zebrafish will be intraperitoneally injected with MPTP 
neurotoxin to induce PD following the procedures explained earlier (Section 3.2.1). Zebrafish will be monitored post injection for any surgical side effects or injuries.

(Corresponding to Objective 3)

3.2.4. Analyses on the effects of HMGB1 knockdown on MPTP-induced zebrafish

Following the knockdown of HMGB1 and induction of MPTP neurotoxin, analyses will be conducted to investigate the effects of HMGB1 knockdown on MPTP-induced zebrafish. Similar analyses will be performed as in Section 3.2.2, which include behavioural, gene expression, and protein expression analyses, the only difference will be, for this section onwards, another group will be added (HMGB1 knocked down group), making a total of three experimental groups, and an additional immunohistochemistry analysis will be conducted as well.

\subsubsection{Behavioural analysis}

The procedures for behavioural analyses will be performed similar to Section 3.2.2.1. All parameters will be analysed statistically to compare the behaviours between normal MPTPinduced, HMGB1-deficient and control groups.

\subsubsection{Gene expression analysis using RT-qPCR}

The procedures for gene expression analysis will be performed similar to Section 3.2.2.2. Comparisons will be made on the targeted gene expressions between normal MPTP-induced, HMGB1-deficient and control groups.

\subsubsection{Protein expression analysis using western blotting}

The procedures for protein expression analysis will be performed similar to Section 3.2.2.3. Comparisons will be made on targeted protein expressions between normal MPTP-induced, HMGB1-deficient and control groups. 


\subsubsection{Immunohistochemistry analysis}

Zebrafish will be anesthetized in $0.1 \%$ tricaine and fixed in $4 \%$ paraformaldehyde (PFA) for 2 days in $4^{\circ} \mathrm{C}$ (Sarath Babu et al., 2016). After that, zebrafish brain will be harvested and stored in phosphate buffered saline (PBS). Brain tissues will be processed and embedded in paraffin wax. Sliced brain tissue sections will be immunostained following standardized in-lab protocols (Sathe et al., 2012).

Samples will be incubated separately with the following primary antibodies: antiHMGB1 antibody (1:1000; Abcam, UK; Cat\# ab233236), anti-glial fibrillary acidic protein antibody (GFAP; 1:1000; Dako, CA, USA; Cat\# Z0334), anti-TH antibody (1:100; Merck Millipore, MA, USA; Cat\# MAB318), and anti-Iba1 antibody (1:100; Wako Pure Chemicals Industries, Germany; Cat\# 019-19741). Correspondingly, anti-rabbit (Cy5-AffiniPure; 1:500; Jackson ImmunoResearch Laboratories, UK; Cat\# 111-175-144) or anti-mouse (Alexa Fluor 488; 1:1000; Thermo Fisher Scientific, MA, USA; Cat\# A-11001) fluorescein-conjugated secondary antibodies will be added for visualization using confocal microscopy.

Immunohistochemistry will be carried out as described and the sections will be counterstained with Nissl (Thionin; Sigma-Aldrich, MO, USA; Cat\# 861340). HMGB1-, TH, GFAP-, Iba1-, and Nissl- positive cells in the ventral diencephalon (zebrafish equivalence of human SNpc) will be counted by blinded examiners using optical fractionator method (Sathe et al., 2012). Ventral telencephalic density of $\mathrm{TH}$ immunoreactivity will be measured as described (Sathe et al., 2012). For double immunofluorescence staining of microglia, sections will be incubated with isolectin B4 conjugated with DyLight 594 (1:200; Vector Laboratories, CA, USA; Cat\# DL-1207) (Lai et al., 2017). The relative expression of each protein in two regions of interest, vDn and vTn, will be compared between groups using Fiji version of ImageJ 1.52p software (Java 1.8.0_172) (NIH, Bethesda, MD) (Sarath Babu et al., 2016). 


\subsection{Statistical analyses}

The obtained results from all analyses will be evaluated statistically using Prism 7.00 for Windows (GraphPad Software, CA, USA). The data will be tabulated and represented as mean (SEM). The statistical significance of the data will be analysed through parametric independent t-test (for two experimental groups) or one-way ANOVA with post-hoc Tukey's test (for three experimental groups). P-values of less than $0.05(p<0.05)$ will be considered as significant for all statistical analyses. All results from this study will be tabulated and/or graphically plotted.

\subsection{Sample size calculation and groupings}

A total of 94 zebrafish will be used in this study (47 males and 47 females), with consideration of $20 \%$ dropouts. Reasons of considering $20 \%$ dropouts is due to possible performance outliers and potential adverse events during surgical procedures (MPTP induction and HMGB1 knockdown).

The sample size of this study is calculated using $G^{*}$ Power software Version 3.1.9.4. (Germany). As this study will be using two types of statistical analyses, two sample sizes are calculated with reference to past literatures. With a $90 \%$ power, $5 \%$ significance level and an effect size of 1.5, the sample sizes required are listed in Table 2. Detailed calculation from G*Power software is attached as Appendix 1.

Based on Table 2, to evaluate Specific Objective 1, zebrafish will be randomly grouped into two groups: 1) Control, and 2) MPTP-induced groups $(n=12)$. Likewise, for Specific Objective 2, zebrafish will be randomly grouped into two groups: 1) Control, and HMGB1deficient groups $(n=12)$, whereas for Specific Objective 3, zebrafish will be randomly allocated to three groups: 1) Control, 2) normal MPTP-induced, and 3) HMGB1-deficient groups $(n=10)$. 
Table 2 Sample size required for each statistical analysis.

\begin{tabular}{|l|l|l|l|l|}
\hline Specific objective & $\begin{array}{l}\text { Statistical } \\
\text { analysis }\end{array}$ & $\begin{array}{l}\text { a Overall } \\
\text { sample size } \\
(n \text { per group })\end{array}$ & $\begin{array}{l}\text { bequired } \\
\text { sample size } \\
(n \text { per group })\end{array}$ & Reference \\
\hline $\begin{array}{l}\text { To establish and validate } \\
\text { zebrafish model of PD via } \\
\text { intraperitoneal injection of } \\
\text { MPTP neurotoxin }\end{array}$ & $\begin{array}{l}\text { Independent } \\
\text { t-test }\end{array}$ & $\begin{array}{l}22(11 \text { per } \\
\text { group })\end{array}$ & $\begin{array}{l}24(12 \text { per } \\
\text { group })\end{array}$ & $\begin{array}{l}\text { (Sarath Babu } \\
\text { et al., 2016) }\end{array}$ \\
\hline $\begin{array}{l}\text { To knockdown the } \\
\text { expression of HMGB1 in } \\
\text { the neurons of zebrafish } \\
\text { brain using anti-sense } \\
\begin{array}{l}\text { HMGB1 morpholino } \\
\text { oligonucleotides. }\end{array}\end{array}$ & $\begin{array}{l}\text { Independent } \\
\text { t-test }\end{array}$ & $\begin{array}{l}22(11 \text { per } \\
\text { group })\end{array}$ & $\begin{array}{l}24(12 \text { per } \\
\text { group })\end{array}$ & $\begin{array}{l}\text { (Zhao } \text { et al., } \\
2011)\end{array}$ \\
\hline $\begin{array}{l}\text { To evaluate the effect of } \\
\text { HMGB1 knockdown at } \\
\text { behavioural, } \\
\text { histopathological, and } \\
\text { molecular levels. }\end{array}$ & $\begin{array}{l}\text { One-way } \\
\text { ANOVA }\end{array}$ & $\begin{array}{l}12(4 \text { per } \\
\text { group })\end{array}$ & $\begin{array}{l}30(10 \text { per } \\
\text { group })\end{array}$ & $\begin{array}{l}\text { (Sarath Babu } \\
\text { et al., 2016) }\end{array}$ \\
\hline
\end{tabular}

${ }^{a}$ Overall sample size is the number of zebrafish needed as calculated by $\mathrm{G}^{*}$ Power software.

${ }^{b}$ Required sample size is the number of zebrafish required to satisfy all analyses (behaviour, RT-qPCR, WB and/or IHC) in a particular Specific Objective. 


\subsection{Flowchart of methodology}

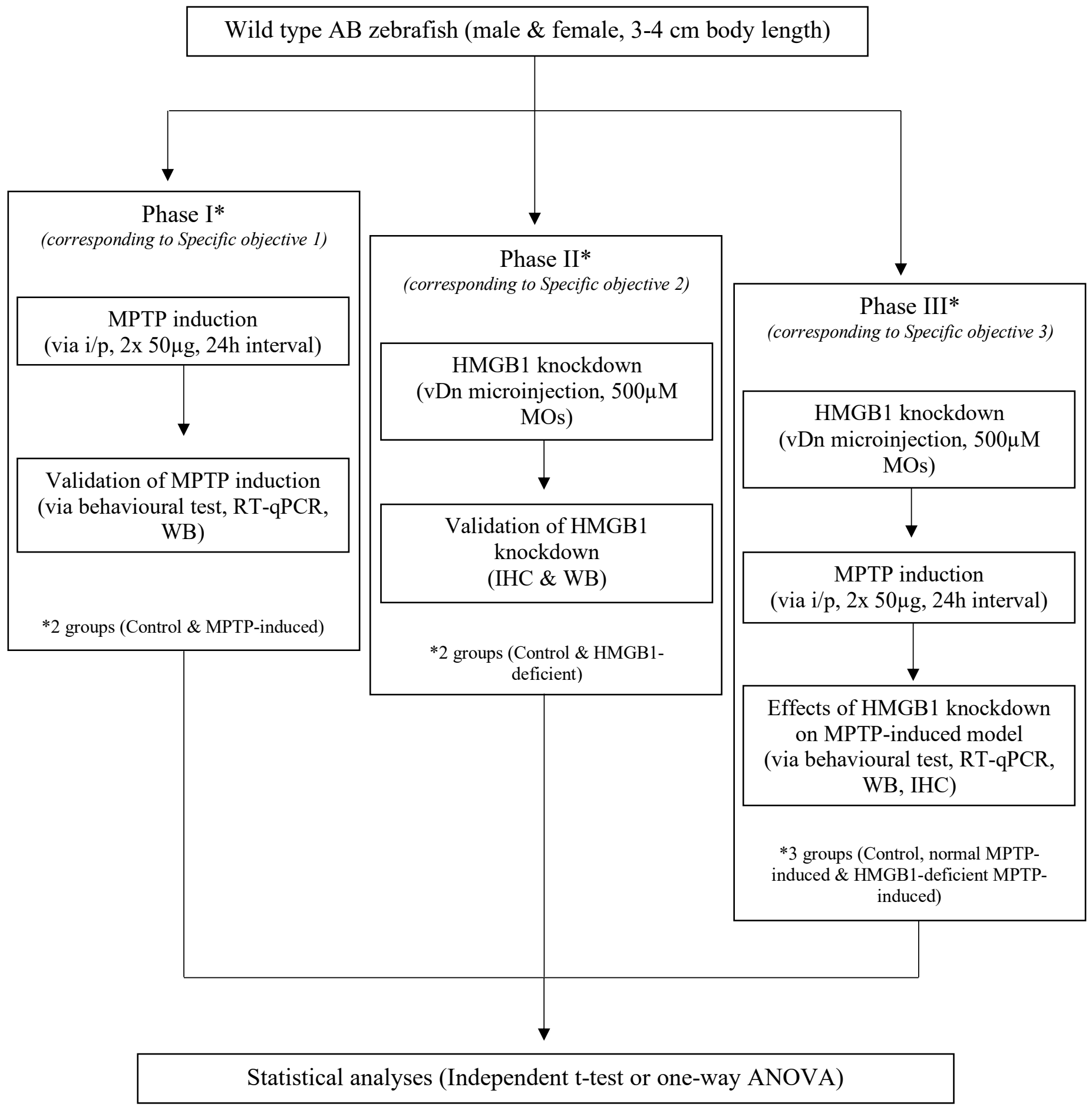




\section{EXPECTED RESULTS}

4.1. Validation of MPTP-induced zebrafish model of PD

\subsubsection{Behavioural analysis}

Graphs representing the performance of the two groups (control and MPTP-induced groups) in behavioural test [parameters: freezing duration (in sec), number of freezing bouts, travelled distance (in $\mathrm{mm}$ ) and swimming speed (in $\mathrm{mm} / \mathrm{s}$ )] will be plotted.

It is expected that MPTP-induced group will have significantly longer freezing duration, higher freezing frequency, shorter travelled distance, and slower swimming speed compared to normal group.

\subsubsection{Gene expression analysis}

A graph representing the relative expression of target genes (lrrk2, park2, park7, sncga and $s n c g b$ genes) obtained from RT-qPCR will be plotted comparing MPTP-induced and control groups.

It is expected that MPTP-induced group will have significantly higher expression levels of all target genes compared to control group.

\subsubsection{Protein expression analysis}

A graph representing the relative expression of target proteins (SNCGA/B, TH, HMGB1, Iba1 and NFkB p65 proteins) obtained from WB will be plotted comparing MPTP-induced and control groups.

It is expected that MPTP-induced group will have significantly higher expression levels of SNCGA/B, HMGB1, Iba1 and NFkB p65 proteins and lower level of TH proteins compared to control group. 
4.2. Validation of HMGB1-knockdown zebrafish

$$
\begin{aligned}
& \text { 4.2.1. Western blot and immunohistochemistry } \\
& \text { analyses }
\end{aligned}
$$

Graphs representing the expression level of HMGB1 protein obtained from WB and IHC will be plotted comparing HMGB1-deficient and control groups.

It is expected that the expression level of HMGB1 protein will be significantly lowered down than normal level following the knockdown, specifically at the targeted region (ventral diencephalon, vDn).

\subsection{Effects of HMGB1-knockdown on MPTP-induced zebrafish model of PD}

\subsubsection{Behavioural analysis}

Graphs representing the performance of the three groups (control, MPTP-induced and HMGB1-deficient groups) in behavioural test [parameters: freezing duration (in sec), number of freezing bouts, travelled distance (in $\mathrm{mm}$ ) and swimming speed (in $\mathrm{mm} / \mathrm{s}$ )] will be plotted.

It is expected that MPTP-induced group will have significantly longer freezing duration, higher freezing frequency, shorter travelled distance, and slower swimming speed compared to control group, while knockdown of HMGB1 will restore the performance back to normal.

\subsubsection{Gene expression analysis}

A graph representing the relative expression of target genes (lrrk2, park2, park7, sncga and $s n c g b$ genes) obtained from RT-qPCR will be plotted comparing control, MPTP-induced and HMGB1-deficient groups.

It is expected that MPTP-induced group will have significantly higher expression levels of all target genes compared to control group, and HMGB1-knockdown will restore the level back to normal. 


\subsubsection{Protein expression analysis}

A graph representing the relative expression of target proteins (SNCGA/B, TH, HMGB1, Iba1 and NFאB p65 proteins) obtained from WB will be plotted comparing MPTP-induced and control groups.

It is expected that MPTP-induced group will have significantly higher expression levels of SNCGA/B, HMGB1, Iba1 and NFkB p65 proteins and lower level of TH proteins compared to control group, while HMGB1-deficient group will have similar expression levels as control.

\subsubsection{Immunohistochemistry analysis}

Graphs representing the expression level of target proteins (HMGB1, GFAP, Iba1, and TH) in specific regions (vDn and vTn) will be plotted comparing the three groups.

It is expected that MPTP-induced group will have significantly higher expression level of HMGB1, GFAP and Iba1, and lower level of TH proteins than control group in both vDn and vTn, while knockdown of HMGB1 will revert the expressions back to normal level. 


\section{REFERENCES}

Aleström, P., D’Angelo, L., Midtlyng, P. J., Schorderet, D. F., Schulte-Merker, S., Sohm, F., \& Warner, S. (2020). Zebrafish: Housing and husbandry recommendations. Laboratory Animals, 54(3), 213-224. https://doi.org/10.1177/0023677219869037

Angelopoulou, E., Piperi, C., \& Papavassiliou, A. G. (2018). High-mobility group box 1 in Parkinson's disease: from pathogenesis to therapeutic approaches. In Journal of Neurochemistry (Vol. 146, Issue 3, pp. 211-218). https://doi.org/10.1111/jnc.14450

Bajpai, P., Sangar, M. C., Singh, S., Tang, W., Bansal, S., Chowdhury, G., Cheng, Q., Fang, J. K., Martin, M. V., Guengerich, F. P., \& Avadhani, N. G. (2013). Metabolism of 1-methyl4-phenyl-1,2,3/6-tetrahydropyridine by mitochondrion-targeted cytochrome P450 2D6 implications in parkinson disease. Journal of Biological Chemistry, 288(6), 4436-4451. https://doi.org/10.1074/jbc.M112.402123

Bajwa, E., Pointer, C. B., \& Klegeris, A. (2019). The role of mitochondrial damage-associated molecular patterns in chronic neuroinflammation. In Mediators of Inflammation (Vol. 2019). https://doi.org/10.1155/2019/4050796

Ball, N., Teo, W.-P., Chandra, S., \& Chapman, J. (2019). Parkinson's Disease and the Environment. Frontiers in Neurology, 10. https://doi.org/10.3389/fneur.2019.00218

Barbazuk, W. B., Korf, I., Kadavi, C., Heyen, J., Tate, S., Wun, E., Bedell, J. A., McPherson, J. D., \& Johnson, S. L. (2000). The syntenic relationship of the zebrafish and human genomes [1]. In Genome Research (Vol. 10, Issue 9, pp. 1351-1358). https://doi.org/10.1101/gr.144700

Barnhill, L. M., Murata, H., \& Bronstein, J. M. (2020). Studying the pathophysiology of Parkinson's disease using zebrafish. In Biomedicines (Vol. 8, Issue 8). https://doi.org/10.3390/BIOMEDICINES8070197

Benchoula, K., Khatib, A., Quzwain, F. M. C., Che Mohamad, C. A., Wan Sulaiman, W. M. A., Wahab, R. A., Ahmed, Q. U., Ghaffar, M. A., Saiman, M. Z., Alajmi, M. F., \& ElSeedi, H. (2019). Optimization of hyperglycemic induction in zebrafish and evaluation of its blood glucose level and metabolite fingerprint treated with psychotria malayana Jack Leaf extract. Molecules, 24(8). https://doi.org/10.3390/molecules24081506

Billingsley, K. J., Bandres-Ciga, S., Saez-Atienzar, S., \& Singleton, A. B. (2018). Genetic risk factors in Parkinson's disease. In Cell and Tissue Research (Vol. 373, Issue 1, pp. 9-20). https://doi.org/10.1007/s00441-018-2817-y

Chen, J. J. (2017). Treatment of psychotic symptoms in patients with Parkinson disease. Mental Health Clinician, 7(6), 262-270. https://doi.org/10.9740/mhc.2017.11.262

Cilia, R., Cereda, E., Akpalu, A., Sarfo, F. S., Cham, M., Laryea, R., Obese, V., Oppon, K., Del Sorbo, F., Bonvegna, S., Zecchinelli, A. L., \& Pezzoli, G. (2020). Natural history of motor symptoms in Parkinson's disease and the long-duration response to levodopa. Brain: A Journal of Neurology, 143(8), 2490-2501. https://doi.org/10.1093/brain/awaa181 
Correia, A. D., Soares, R. S., Rahimi, K., Sousa, S., \& Outeiro, T. F. (2017). Green Fluorescent Protein Labeling of Dopaminergic Neurons in Zebrafish for the Study of Parkinson's Diseases. Journal of Microbiology \& Experimentation, 4(1). https://doi.org/10.15406/jmen.2017.04.00101

DeMaagd, G., \& Philip, A. (2015). Parkinson's disease and its management part 1: Disease entity, risk factors, pathophysiology, clinical presentation, and diagnosis. Pharmacy and Therapeutics, 40(8), 504-532.

Eisen, J. S. (2020). History of Zebrafish Research. In The Zebrafish in Biomedical Research (pp. 3-14). https://doi.org/10.1016/b978-0-12-812431-4.00001-4

Fang, P., Pan, H. C., Lin, S. L., Zhang, W. Q., Rauvala, H., Schachner, M., \& Shen, Y. Q. (2014). HMGB1 contributes to regeneration after spinal cord injury in adult zebrafish. In Molecular Neurobiology (Vol. 49, Issue 1, pp. 472-483). https://doi.org/10.1007/s12035013-8533-4

Fang, P., Pan, H. C., Lin, S. L., Zhang, W. Q., Rauvala, H., Schachner, M., \& Shen, Y. Q. (2014). HMGB1 contributes to regeneration after spinal cord injury in adult zebrafish. In Molecular Neurobiology (Vol. 49, Issue 1, pp. 472-483). https://doi.org/10.1007/s12035013-8533-4

Feigin, V. L., Krishnamurthi, R. V., Theadom, A. M., Abajobir, A. A., Mishra, S. R., Ahmed, M. B., Abate, K. H., Mengistie, M. A., Wakayo, T., Abd-Allah, F., Abdulle, A. M., Abera, S. F., Mohammed, K. E., Abyu, G. Y., Asgedom, S. W., Atey, T. M., Betsu, B. D., Mezgebe, H. B., Tuem, K. B., ... Zaki, M. E. (2017). Global, regional, and national burden of neurological disorders during 1990-2015: a systematic analysis for the Global Burden of Disease Study 2015. The Lancet Neurology, 16(11), 877-897. https://doi.org/10.1016/S1474-4422(17)30299-5

Fernagut, P. O. (2016). Etiology and Progression of Parkinson's Disease: Cross-Talk Between Environmental Factors and Genetic Vulnerability. In Handbook of Behavioral Neuroscience (Vol. 24, pp. 803-819). https://doi.org/10.1016/B978-0-12-802206$1.00040-4$

Frank, M. G., Weber, M. D., Watkins, L. R., \& Maier, S. F. (2015). Stress sounds the alarmin: The role of the danger-associated molecular pattern HMGB1 in stress-induced neuroinflammatory priming. In Brain, Behavior, and Immunity (Vol. 48, pp. 1-7). https://doi.org/10.1016/j.bbi.2015.03.010

Freeman, J. L., Adeniyi, A., Banerjee, R., Dallaire, S., Maguire, S. F., Chi, J., Ng, B. L., Zepeda, C., Scott, C. E., Humphray, S., Rogers, J., Zhou, Y., Zon, L. I., Carter, N. P., Yang, F., \& Lee, C. (2007). Definition of the zebrafish genome using flow cytometry and cytogenetic mapping. BMC Genomics, 8. https://doi.org/10.1186/1471-2164-8-195

Gao, H. M., Zhou, H., Zhang, F., Wilson, B. C., Kam, W., \& Hong, J. S. (2011). HMGB1 acts on microglia Mac1 to mediate chronic neuroinflammation that drives progressive neurodegeneration. Journal of Neuroscience, 31(3), 1081-1092. https://doi.org/10.1523/JNEUROSCI.3732-10.2011 
Hajj, R. (2018). Parkinson disease therapies and drugs. In Pathology, Prevention and Therapeutics of Neurodegenerative Disease (pp. 151-158). https://doi.org/10.1007/978981-13-0944-1_13

Harris, J. P., Burrell, J. C., Struzyna, L. A., Chen, H. I., Serruya, M. D., Wolf, J. A., Duda, J. E., \& Cullen, D. K. (2020). Emerging regenerative medicine and tissue engineering strategies for Parkinson's disease. In npj Parkinson's Disease (Vol. 6, Issue 1). https://doi.org/10.1038/s41531-019-0105-5

Hermanowicz, N., Jones, S. A., \& Hauser, R. A. (2019). Impact of non-motor symptoms in parkinson's disease: A PMDAlliance survey. Neuropsychiatric Disease and Treatment, 15, 2205-2212. https://doi.org/10.2147/NDT.S213917

Hickey, P., \& Stacy, M. (2016). Deep brain stimulation: A paradigm shifting approach to treat Parkinson's disease. In Frontiers in Neuroscience (Vol. 10, Issue APR). https://doi.org/10.3389/fnins.2016.00173

Hindle, J. V. (2010). Ageing, neurodegeneration and Parkinson's disease. In Age and Ageing (Vol. 39, Issue 2, pp. 156-161). https://doi.org/10.1093/ageing/afp223

Howe, K., Clark, M. D., Torroja, C. F., Torrance, J., Berthelot, C., Muffato, M., Collins, J. E., Humphray, S., McLaren, K., Matthews, L., McLaren, S., Sealy, I., Caccamo, M., Churcher, C., Scott, C., Barrett, J. C., Koch, R., Rauch, G. J., White, S., ... Stemple, D. L. (2013). The zebrafish reference genome sequence and its relationship to the human genome. Nature, 496(7446), 498-503. https://doi.org/10.1038/nature12111

Huang, J., Yang, J., Shen, Y., Jiang, H., Han, C., Zhang, G., Liu, L., Xu, X., Li, J., Lin, Z., Xiong, N., Zhang, Z., Xiong, J., \& Wang, T. (2017). HMGB1 mediates autophagy dysfunction via perturbing beclin1-Vps34 complex in dopaminergic cell model. Frontiers in Molecular Neuroscience, 10. https://doi.org/10.3389/fnmol.2017.00013

Ketzef, M., Spigolon, G., Johansson, Y., Bonito-Oliva, A., Fisone, G., \& Silberberg, G. (2017). Dopamine Depletion Impairs Bilateral Sensory Processing in the Striatum in a PathwayDependent Manner. Neuron, 94(4), 855-865.e5. https://doi.org/10.1016/j.neuron.2017.05.004

Kleinhappel, T. K., Pike, T. W., \& Burman, O. H. P. (2019). Stress-induced changes in group behaviour. Scientific Reports, 9(1). https://doi.org/10.1038/s41598-019-53661-w

Koster, R., \& Sassen, W. A. (2015). A molecular toolbox for genetic manipulation of zebrafish. Advances in Genomics and Genetics, 151. https://doi.org/10.2147/agg.s57585

Kouli, A., Torsney, K. M., \& Kuan, W.-L. (2018). Parkinson's Disease: Etiology, Neuropathology, and Pathogenesis. In Parkinson's Disease: Pathogenesis and Clinical Aspects (pp.

3-26). https://doi.org/10.15586/codonpublications.parkinsonsdisease.2018.ch1

Krishna, R., Ali, M., \& Moustafa, A. A. (2014). Effects of combined MAO-B inhibitors and levodopa vs monotherapy in Parkinson's disease. Frontiers in Aging Neuroscience, 6(JUL). https://doi.org/10.3389/fnagi.2014.00180 
Lai, S. L., Marín-Juez, R., Moura, P. L., Kuenne, C., Lai, J. K. H., Tsedeke, A. T., Guenther, S., Looso, M., \& Stainier, D. Y. R. (2017). Reciprocal analyses in zebrafish and medaka reveal that harnessing the immune response promotes cardiac regeneration. ELife, 6. https://doi.org/10.7554/eLife.25605

Lam, C. S., Korzh, V., \& Strahle, U. (2005). Zebrafish embryos are susceptible to the dopaminergic neurotoxin MPTP. European Journal of Neuroscience, 21(6), 1758-1762. https://doi.org/10.1111/j.1460-9568.2005.03988.x

Langston, J. W. (2017). The MPTP story. In Journal of Parkinson's Disease (Vol. 7, pp. S11S19). https://doi.org/10.3233/JPD-179006

Lee, S., Nam, Y., Koo, J. Y. oun., Lim, D., Park, J., Ock, J., Kim, J., Suk, K., \& Park, S. B. u. (2014). A small molecule binding HMGB1 and HMGB2 inhibits microglia-mediated neuroinflammation. Nature Chemical Biology, 10(12), 1055-1060. https://doi.org/10.1038/nchembio.1669

Lee, S., Nam, Y., Koo, J. Y. oun., Lim, D., Park, J., Ock, J., Kim, J., Suk, K., \& Park, S. B. u. (2014). A small molecule binding HMGB1 and HMGB2 inhibits microglia-mediated neuroinflammation. Nature Chemical Biology, 10(12), 1055-1060. https://doi.org/10.1038/nchembio.1669

Magna, M., \& Pisetsky, D. S. (2014). The role of HMGB1 in the pathogenesis of inflammatory and autoimmune diseases. Molecular Medicine, 20(1), 138-146. https://doi.org/10.2119/molmed.2013.00164

Maiti, P., Manna, J., Dunbar, G. L., Maiti, P., \& Dunbar, G. L. (2017). Current understanding of the molecular mechanisms in Parkinson's disease: Targets for potential treatments. In Translational Neurodegeneration (Vol. 6, Issue 1). https://doi.org/10.1186/s40035-0170099-z

Meade, R. M., Fairlie, D. P., \& Mason, J. M. (2019). Alpha-synuclein structure and Parkinson's disease - Lessons and emerging principles. In Molecular Neurodegeneration (Vol. 14, Issue 1). https://doi.org/10.1186/s13024-019-0329-1

Mimaki, M., Wang, X., McKenzie, M., Thorburn, D. R., \& Ryan, M. T. (2012). Understanding mitochondrial complex I assembly in health and disease. In Biochimica et Biophysica Acta - Bioenergetics (Vol. 1817, Issue 6, pp. 851-862). https://doi.org/10.1016/j.bbabio.2011.08.010

Morales, I., Farías, G. A., Cortes, N., \& Maccioni, R. B. (2016). Neuroinflammation and Neurodegeneration. In Update on Dementia. https://doi.org/10.5772/64545

Moustafa, A. A., Chakravarthy, S., Phillips, J. R., Gupta, A., Keri, S., Polner, B., Frank, M. J., \& Jahanshahi, M. (2016). Motor symptoms in Parkinson's disease: A unified framework. In Neuroscience and Biobehavioral Reviews (Vol. 68, pp. 727-740). https://doi.org/10.1016/j.neubiorev.2016.07.010

Murugesu, S., Khatib, A., Ahmed, Q. U., Ibrahim, Z., Uzir, B. F., Benchoula, K., Yusoff, N. I. N., Perumal, V., Alajmi, M. F., Salamah, S., \& El-Seedi, H. R. (2019). Toxicity study on Clinacanthus nutans leaf hexane fraction using Danio rerio embryos. Toxicology Reports, 6, 1148-1154. https://doi.org/10.1016/j.toxrep.2019.10.020 
Pang, S. Y. Y., Ho, P. W. L., Liu, H. F., Leung, C. T., Li, L., Chang, E. E. S., Ramsden, D. B., \& Ho, S. L. (2019). The interplay of aging, genetics and environmental factors in the pathogenesis of Parkinson's disease. In Translational Neurodegeneration (Vol. 8, Issue 1). https://doi.org/10.1186/s40035-019-0165-9

Paudel, Y. N., Shaikh, M. F., Chakraborti, A., Kumari, Y., Aledo-Serrano, Á., Aleksovska, K., Alvim, M. K. M. H., \& Othman, I. (2018). HMGB1: A common biomarker and potential target for TBI, neuroinflammation, epilepsy, and cognitive dysfunction. In Frontiers in Neuroscience (Vol. 12, Issue SEP). https://doi.org/10.3389/fnins.2018.00628

Peball, M., Krismer, F., Knaus, H. G., Djamshidian, A., Werkmann, M., Carbone, F., Ellmerer, P., Heim, B., Marini, K., Valent, D., Goebel, G., Ulmer, H., Stockner, H., Wenning, G. K., Stolz, R., Krejcy, K., Poewe, W., \& Seppi, K. (2020). Non-Motor Symptoms in Parkinson's Disease are Reduced by Nabilone. Annals of Neurology, 88(4), 712-722. https://doi.org/10.1002/ana.25864

Perier, C., \& Vila, M. (2012). Mitochondrial biology and Parkinson's disease. Cold Spring Harbor Perspectives in Medicine, 2(2). https://doi.org/10.1101/cshperspect.a009332

Ray Dorsey, E., Elbaz, A., Nichols, E., Abd-Allah, F., Abdelalim, A., Adsuar, J. C., Ansha, M. G., Brayne, C., Choi, J. Y. J., Collado-Mateo, D., Dahodwala, N., Do, H. P., Edessa, D., Endres, M., Fereshtehnejad, S. M., Foreman, K. J., Gankpe, F. G., Gupta, R., Hankey, G. J., .. Murray, C. J. L. (2018). Global, regional, and national burden of Parkinson's disease, 1990-2016: a systematic analysis for the Global Burden of Disease Study 2016. The Lancet Neurology, 17(11), 939-953. https://doi.org/10.1016/S1474-4422(18)302953

Reeve, A., Simcox, E., \& Turnbull, D. (2014). Ageing and Parkinson's disease: Why is advancing age the biggest risk factor? In Ageing Research Reviews (Vol. 14, Issue 1, pp. 19-30). https://doi.org/10.1016/j.arr.2014.01.004

Robea, M. A., Balmus, I. M., Ciobica, A., Strungaru, S., Plavan, G., Gorgan, L. D., Savuca, A., \& Nicoara, M. (2020). Parkinson's Disease-Induced Zebrafish Models: Focussing on Oxidative Stress Implications and Sleep Processes. In Oxidative Medicine and Cellular Longevity (Vol. 2020). https://doi.org/10.1155/2020/1370837

Santoro, M., Maetzler, W., Stathakos, P., Martin, H. L., Hobert, M. A., Rattay, T. W., Gasser, T., Forrester, J. V., Berg, D., Tracey, K. J., Riedel, G., \& Teismann, P. (2016). In-vivo evidence that high mobility group box 1 exerts deleterious effects in the 1-methyl-4phenyl-1,2,3,6-tetrahydropyridine model and Parkinson's disease which can be attenuated by glycyrrhizin. Neurobiology of Disease, 91, 59-68. https://doi.org/10.1016/j.nbd.2016.02.018

Santoro, M., Maetzler, W., Stathakos, P., Martin, H. L., Hobert, M. A., Rattay, T. W., Gasser, T., Forrester, J. V., Berg, D., Tracey, K. J., Riedel, G., \& Teismann, P. (2016). In-vivo evidence that high mobility group box 1 exerts deleterious effects in the 1-methyl-4phenyl-1,2,3,6-tetrahydropyridine model and Parkinson's disease which can be attenuated by glycyrrhizin. Neurobiology of Disease, 91, 59-68. https://doi.org/10.1016/j.nbd.2016.02.018 
Sarath Babu, N., Murthy, C. L. N., Kakara, S., Sharma, R., Brahmendra Swamy, C. V., \& Idris, M. M. (2016). 1-Methyl-4-phenyl-1,2,3,6-tetrahydropyridine induced Parkinson's disease in zebrafish. Proteomics, 16(9), 1407-1420. https://doi.org/10.1002/pmic.201500291

Sasaki, T., Liu, K., Agari, T., Yasuhara, T., Morimoto, J., Okazaki, M., Takeuchi, H., Toyoshima, A., Sasada, S., Shinko, A., Kondo, A., Kameda, M., Miyazaki, I., Asanuma, M., Borlongan, C. V., Nishibori, M., \& Date, I. (2016). Anti-high mobility group box 1 antibody exerts neuroprotection in a rat model of Parkinson's disease. Experimental Neurology, 275, 220-231. https://doi.org/10.1016/j.expneurol.2015.11.003

Sathe, K., Maetzler, W., Lang, J. D., Mounsey, R. B., Fleckenstein, C., Martin, H. L., Schulte, C., Mustafa, S., Synofzik, M., Vukovic, Z., Itohara, S., Berg, D., \& Teismann, P. (2012). $\mathrm{S} 100 \mathrm{~B}$ is increased in Parkinson's disease and ablation protects against MPTP-induced toxicity through the RAGE and TNF- $\alpha$ pathway. Brain, 135(11), 3336-3347. https://doi.org/10.1093/brain/aws250

Shehwana, H., \& Konu, O. (2019). Comparative Transcriptomics Between Zebrafish and Mammals: A Roadmap for Discovery of Conserved and Unique Signaling Pathways in Physiology and Disease. Frontiers in Cell and Developmental Biology, 7. https://doi.org/10.3389/fcell.2019.00005

Sochocka, M., Diniz, B. S., \& Leszek, J. (2017). Inflammatory Response in the CNS: Friend or Foe? In Molecular Neurobiology (Vol. 54, Issue 10, pp. 8071-8089). https://doi.org/10.1007/s12035-016-0297-1

Stefanis, L. (2012). $\alpha$-Synuclein in Parkinson's disease. Cold Spring Harbor Perspectives in Medicine, 2(2). https://doi.org/10.1101/cshperspect.a009399

Stepens, A., \& Taba, P. (2016). The Neuropathology of Movement Disorders in 1-Methyl-4Phenyl-1,2,3,6-Tetrahydropyridine (MPTP) and Methcathinone (Ephedrone) Addictions. In Neuropathology of Drug Addictions and Substance Misuse (Vol. 3, pp. 924-934). https://doi.org/10.1016/B978-0-12-800634-4.00091-3

Sveinbjornsdottir, S. (2016). The clinical symptoms of Parkinson's disease. In Journal of Neurochemistry (pp. 318-324). https://doi.org/10.1111/jnc.13691

Toledo-Ibarra, G. A., Rojas-Mayorquín, A. E., \& Girón-Pérez, M. I. (2013). Influence of the cholinergic system on the immune response of teleost fishes: Potential model in biomedical research. In Clinical and Developmental Immunology (Vol. 2013). https://doi.org/10.1155/2013/536534

Tran, J., Anastacio, H., \& Bardy, C. (2020). Genetic predispositions of Parkinson's disease revealed in patient-derived brain cells. In npj Parkinson's Disease (Vol. 6, Issue 1). https://doi.org/10.1038/s41531-020-0110-8

Troncoso-Escudero, P., Parra, A., Nassif, M., \& Vidal, R. L. (2018). Outside in: Unraveling the role of neuroinflammation in the progression of Parkinson's disease. In Frontiers in Neurology (Vol. 9, Issue OCT). https://doi.org/10.3389/fneur.2018.00860

Vaz, R. L., Outeiro, T. F., \& Ferreira, J. J. (2018). Zebrafish as an animal model for drug discovery in Parkinson's disease and other movement disorders: A systematic review. In Frontiers in Neurology (Vol. 9, Issue JUN). https://doi.org/10.3389/fneur.2018.00347 
Vijayanathan, Y., Lim, F. T., Lim, S. M., Long, C. M., Tan, M. P., Majeed, A. B. A., \& Ramasamy, K. (2017). 6-OHDA-Lesioned Adult Zebrafish as a Useful Parkinson's Disease Model for Dopaminergic Neuroregeneration. Neurotoxicity Research, 32(3), 496-508. https://doi.org/10.1007/s12640-017-9778-x

Wang, Q., Liu, Y., \& Zhou, J. (2015). Neuroinflammation in Parkinson's disease and its potential as therapeutic target. In Translational Neurodegeneration (Vol. 4, Issue 1). https://doi.org/10.1186/s40035-015-0042-0

Wasel, O., \& Freeman, J. L. (2020). Chemical and genetic zebrafish models to define mechanisms of and treatments for dopaminergic neurodegeneration. International Journal of Molecular Sciences, 21(17), 1-14. https://doi.org/10.3390/ijms21175981

Yang, H., Antoine, D. J., Andersson, U., \& Tracey, K. J. (2013). The many faces of HMGB1: molecular structure-functional activity in inflammation, apoptosis, and chemotaxis. Journal of Leukocyte Biology, 93(6), 865-873. https://doi.org/10.1189/jlb.1212662

Yang, Y., Tang, B. S., \& Guo, J. F. (2016). Parkinson's disease and cognitive impairment. In Parkinson's Disease (Vol. 2016). https://doi.org/10.1155/2016/6734678

Yuan, S., Zhao, L., \& Sun, Z. (2013). Dissecting the functional interplay between the TOR pathway and the cilium in zebrafish. In Methods in Enzymology (Vol. 525, pp. 159-189). https://doi.org/10.1016/B978-0-12-397944-5.00009-2

Zawada, W. M., Banninger, G. P., Thornton, J., Marriott, B., Cantu, D., Rachubinski, A. L., Das, M., Griffin, W. S. T., \& Jones, S. M. (2011). Generation of reactive oxygen species in 1-methyl-4-phenylpyridinium (MPP+) treated dopaminergic neurons occurs as an NADPH oxidase-dependent two-wave cascade. Journal of Neuroinflammation, 8. https://doi.org/10.1186/1742-2094-8-129

Zeng, X. S., Geng, W. S., \& Jia, J. J. (2018). Neurotoxin-Induced Animal Models of Parkinson Disease: Pathogenic Mechanism and Assessment. In ASN Neuro (Vol. 10). https://doi.org/10.1177/1759091418777438

Zhao, X., Kuja-Panula, J., Rouhiainen, A., Chen, Y. C., Panula, P., \& Rauvala, H. (2011). High mobility group box-1 (HMGB1; amphoterin) is required for zebrafish brain development. Journal of Biological Chemistry, 286(26), 23200-23213. https://doi.org/10.1074/jbc.M111.223834 


\section{GANTT CHART AND MILESTONES}

\subsection{Gantt chart}

\begin{tabular}{|c|c|c|c|c|c|c|c|c|c|c|c|c|c|c|c|c|c|c|c|c|c|c|}
\hline Year & 202 & & 20 & & & & & & & & & & & & 20 & & & & & & & \\
\hline Activity/month & $\mathrm{N}$ & $\mathrm{D}$ & $\mathrm{J}$ & $\mathrm{F}$ & $\mathrm{M}$ & A & $\mathrm{M}$ & $\mathrm{J}$ & $\mathrm{J}$ & A & $S$ & $\mathrm{O}$ & $\mathrm{N}$ & D & $\mathrm{J}$ & $\mathrm{F}$ & $\mathrm{M}$ & A & $\mathrm{M}$ & $\mathrm{J}$ & $\mathrm{J}$ & A \\
\hline $\begin{array}{l}\text { Proposal defence } \\
\text { and ethics approval }\end{array}$ & & & & & & & & & & & & & & & & & & & & & & \\
\hline $\begin{array}{l}\text { Breeding and } \\
\text { maintenance of } \\
\text { zebrafish }\end{array}$ & & & & & & & & & & & & & & & & & & & & & & \\
\hline $\begin{array}{l}\text { Induction of MPTP } \\
\text { via } 1 / p \text { injection }\end{array}$ & & & & & & & & & & & & & & & & & & & & & & \\
\hline $\begin{array}{l}\text { Validation of } \\
\text { MPTP-induced } \\
\text { model }\end{array}$ & & & & & & & & & & & & & & & & & & & & & & \\
\hline $\begin{array}{l}\text { HMGB1 } \\
\text { knockdown via } \\
\text { MOs microinjectiol }\end{array}$ & & & & & & & & & & & & & & & & & & & & & & \\
\hline $\begin{array}{l}\text { Validation of } \\
\text { HMGB1-deficient } \\
\text { model }\end{array}$ & & & & & & & & & & & & & & & & & & & & & & \\
\hline $\begin{array}{l}\text { Induction of MPTP } \\
\text { on HMGB1- } \\
\text { knocked down } \\
\text { model }\end{array}$ & & & & & & & & & & & & & & & & & & & & & & \\
\hline $\begin{array}{l}\text { Analyses on the } \\
\text { effects of HMGB1 } \\
\text { knockdown on } \\
\text { MPTP-induced } \\
\text { model }\end{array}$ & & & & & & & & & & & & & & & & & & & & & & \\
\hline $\begin{array}{l}\text { Statistical analyses } \\
\text { and report writing }\end{array}$ & & & & & & & & & & & & & & & & & & & & & & \\
\hline
\end{tabular}

\subsection{Milestones}

\begin{tabular}{|l|c|c|}
\hline \multicolumn{1}{|c|}{ Completed task } & Completion date & Percentage of completion (\%) \\
\hline $\begin{array}{l}\text { Induced MPTP on zebrafish via } \\
\text { i/p injection }\end{array}$ & $31^{\text {st }}$ May 2021 & $10 \%$ \\
\hline $\begin{array}{l}\text { Validated MPTP-induced } \\
\text { zebrafish model }\end{array}$ & $31^{\text {st }}$ July 2021 & $30 \%$ \\
\hline $\begin{array}{l}\text { Knocked down HMGB1 on } \\
\text { zebrafish via MOs microinjection }\end{array}$ & $30^{\text {th }}$ September 2021 & $40 \%$ \\
\hline $\begin{array}{l}\text { Validated HMGB1-deficient } \\
\text { zebrafish model }\end{array}$ & $30^{\text {th }}$ November 2021 \\
\hline $\begin{array}{l}\text { Induced MPTP on HMGB1- } \\
\text { deficient zebrafish model }\end{array}$ & $28^{\text {th }}$ February 2022 \\
\hline $\begin{array}{l}\text { Analysed the effects of HMGB1 } \\
\text { knockdown on MPTP-induced } \\
\text { zebrafish model }\end{array}$ & 30 th June 2022 & $70 \%$ \\
\hline $\begin{array}{l}\text { Completion of statistical analyses } \\
\text { and report writing }\end{array}$ & $31^{\text {st }}$ August 2022 & $100 \%$ \\
\hline
\end{tabular}




\section{APPENDIX 1}

a)

$\begin{array}{lll}\mathrm{t} \text { tests - Means: Difference between two independent means (two groups) } \\ \text { Analysis: } \quad \text { A priori: Compute required sample } & \text { size } \\ \text { Input: } & \text { Tail(s) } & =\text { Two } \\ & \text { Effect size d } & =1.5 \\ & \alpha \text { err prob } & =0.05 \\ & \text { Power }(1-\beta \text { err prob) } & =0.9 \\ & \text { Allocation ratio N2/N1 } & =1 \\ \text { Output: } & \text { Noncentrality parameter } \delta & =3.5178118 \\ & \text { Critical t } & =2.0859634 \\ & \text { Df } & =20 \\ & \text { Sample size group 1 } & =11 \\ & \text { Sample size group 2 } & =11 \\ & \text { Total sample size } & =22 \\ & \text { Actual power } & =0.9168991\end{array}$

b)

Thursday, November 26, $2020--71.05: 38$

F tests - ANOVA: Fixed effects, omnibus, one-way

Analysis: A priori: Compute required sample size

Input: $\quad$ Effect size $f \quad=1.5$

$\alpha$ err prob $\quad=0.05$

Power $(1-\beta$ err prob) $\quad=0.9$

Number of groups $=3$

Output: $\quad$ Noncentrality parameter $\lambda \quad=27.0000000$

Critical F $\quad=4.2564947$

Numerator df $\quad=2$

Denominator $\mathrm{df} \quad=9$

Total sample size $\quad=12$

Actual power $\quad=0.9785871$

Sample size for each statistical method as calculated by G*Power software. a) independent t-test for Specific Objective 1 and 2, b) One-way ANOVA for Specific Objective 3. 